Krzysztof Ruchniewicz

\title{
Drei Daten, drei Anfänge
}

\author{
Das polnische Dilemma
}

Der 100. Jahrestag der Wiedererlangung der Unabhängigkeit Polens im November dieses Jahrs wird zu verschiedenen Resümees anregen und mögliche Entwicklungswege für die Zukunft aufzeigen. Besonders auffällig sind in diesem vergangenen Jahrhundert aus der Perspektive der polnischen und europäischen Geschichte drei Daten. Jedes dieser Daten war bahnbrechend, was unter Historikern unstrittig ist. Das erste stand im Zusammenhang mit dem Ende des Ersten Weltkriegs und der Entstehung neuer Staaten in Mittel- und Osteuropa, darunter Polen. Zum ersten Mal seit mehr als 100 Jahren wurde die Karte dieses Teils Europas neu gezeichnet. In Mittel- und Osteuropa entstanden neue Nationalstaaten. Das zweite Datum hing mit dem Ende des Zweiten Weltkriegs und schließlich das dritte mit dem Zusammenbruch des Kommunismus in Ostmitteleuropa zusammen.

\section{Drei Meilensteine im Hintergrund}

Das Jahr 1918 markiert den Beginn einer neuen Ära, deren Form und Gestalt sehr fragil war und mit zahlreichen Belastungen einherging. Das wiedergeborene Polen sah sich mit vier allgemeinen Problemen konfrontiert, die trotz der inzwischen vergangenen vielen Jahrzehnte und wechselnder geopolitischer Konstellationen bis heute nichts an Bedeutung verloren haben. Diese waren: die Suche nach einem Modus Vivendi mit seinen Nachbarn als unabhängiger Staat, insbesondere mit Deutschland und Russland (später der UdSSR), die Verringerung der wirtschaftlichen Distanz zu den entwickelteren europäischen Ländern, die Überwindung der Spaltungen in der Gesellschaft, um potenzielle Ausbrüche von Unzufriedenheit zu vermeiden, und die Förderung von Aktivitäten zur Verteidigung Polens und seiner Entwicklung sowie schließlich eine endgültige Bestimmung der Identität des Staats und seiner Bürger.

Das Jahr 1918 ermöglichte es Polen, erneut auf der politischen Landkarte Europas zu erscheinen und politische, wirtschaftliche sowie kulturelle Grundlagen für eine unabhängige staatliche Existenz zu schaffen. Es war die Erfüllung der Unabhängigkeitsprogramme und -bemühungen vieler Generationen. Nach mehr als 100 Jahren konnte sich Polen als unabhängiger Staat entwickeln, in dessen Grenzen die überwiegende Mehrheit der Polen (mit Ausnahme der polnischen Minderheiten, die innerhalb der Grenzen Deutschlands und der UdSSR verblieben) und mehrere nationale Minderheiten lebten.

Das zweite dieser Daten ist das Jahr 1945, als nicht nur der Zweite Weltkrieg zu Ende ging, sondern auch die Länder Mittel- und Osteuropas, darunter Polen, in 
die Zone des politischen, wirtschaftlichen und kulturellen Einflusses der UdSSR gerieten. Infolge des Zweiten Weltkriegs erlitt Polen enorme personelle und materielle Verluste, verlor die Hälfte seines Vorkriegsgebiets und erhielt als Entschädigung ehemals deutsche Gebiete östlich von Oder und Neiße.

Die Unterordnung Polens unter die UdSSR schloss es jahrzehntelang aus dem freien Europa aus, seine politische und wirtschaftliche Aktivität beschränkte sich auf die Länder des Ostblocks. Gab es dennoch Verbindungen mit der Zeit vor 1939? Haben sich die Kommunisten irgendwie auf die politischen Gedanken der Vorkriegspolitiker bezogen? Erwähnenswert sind hier die Ausführungen von Jerzy Holzer, einem Experten für die Geschichte Europas und Polens im 20. Jahrhundert. Seiner Meinung nach überlebte das politische Denken Roman Dmowskis oder Józef Piłsudskis bei der Führung der kommunistischen Partei in größerem Umfang als in den Kreisen der antikommunistischen Opposition und beeinflusste durch die Propaganda der staatlichen Behörden das gesellschaftliche Bewusstsein. Das bedeutet natürlich nicht, dass die Kommunisten diese Personen ausdrücklich als ideologische Schirmherren bezeichnet haben. „Schließlich waren es die Machthaber der Volksrepublik Polen, die ständig über die Einheit der Nation sprachen“, schrieb Holzer, „über die Verteidigung seiner nationalen Interessen gegen Feinde, das war der Topos der deutschen, der Topos der jüdischen Bedrohung, und sogar viele Akzente einer Bedrohung durch den ukrainischen Nationalismus, mit der Legende vom Kampf im Bieszczady-Gebirge“. Und auf Piłsudski, auf die „Vorstellung vom Staat als obersten Wert“, schlussfolgerte der polnische Forscher, ,auf seine Rolle als Oberhaupt sowie auf die spezifische Mission der Armee, bezog sich eindeutig Wojciech Jaruzelski nach der Einführung des Kriegsrechts". ${ }^{1}$

Das Jahr 1989, das dritte der erwähnten Daten, der „Völkerherbst“, markierte den Beginn der Auflösung der UdSSR und die Wiedererlangung der Souveränität und Unabhängigkeit Mittel- und Osteuropas. Die Karte Europas musste wiederum neu gezeichnet werden. Der Zusammenbruch der UdSSR vertiefte diesen Prozess mit der Entstehung der unabhängigen Staaten Ukraine, Weißrussland, Lettland, Litauen und Estland. Die Tschechoslowakei teilte sich am 1. Januar 1993 in zwei Staaten auf. Im Falle Polens war der wichtigste Effekt der Veränderungen die Beendigung der negativen geopolitischen Auswirkungen seiner Lage zwischen Deutschland und der UdSSR (jetzt Russland).

Deutschland vereinigte sich und stärkte seine europäische und demokratische Entwicklung. Russland hingegen befindet sich seit vielen Jahren in einem Zustand innerer Schwäche. Mit dem Abschluss des Vertragswerks zwischen Polen

\footnotetext{
${ }^{1}$ Polityka vom 12.12.2005: „Przyszłość mądrzejsza od historii. O polskiej geografii politycznej, o Piłsudskim i Dmowskim, o sanacji i Solidarności z profesorem Jerzym Holzerem rozmawia Wiesław Władyka“; www.polityka.pl/tygodnikpolityka/spoleczenstwo/166194,1,jak-rozumiec-polska-przeszlosc.read [4.2.2018]. Das Bieszczady-Gebirge wurde nach dem Zweiten Weltkrieg zum Schauplatz der Kämpfe zwischen der Polnischen Armee und Ukrainischen Aufständischen Armee. 1947 führte die polnische kommunistische Regierung die sogenannte Aktion-Weichsel durch, in deren Folge ca. 140.000 Ukrainer in die neuen Westgebiete Polens deportiert wurden.
} 
und Deutschland 1990/91 wurde der sogenannte Fatalismus der Feindschaft beendet. Polen entschloss sich bald zu einem Beitritt zur North Atlantic Treaty Organization (NATO) und Europäischen Union (EU). Der schmerzhafte Prozess des wirtschaftlichen, politischen und sozialen Wandels, der nicht nur eine Voraussetzung für den Beitritt zur EU, sondern schlicht eine notwendige Modernisierung darstellte, hat Polen und einigen anderen postkommunistischen Ländern neue Perspektiven eröffnet.

\section{Grundprobleme der Zwischenkriegszeit}

Es war notwendig, diesen Hintergrund anhand von drei Meilensteinen aus dem letzten Jahrhundert zu vergegenwärtigen, um die gegenwärtige Herangehensweise zur ersten Epoche, der Zwischenkriegszeit, besser zu verstehen. Eine der Folgen des Zusammenbruchs der kommunistischen Macht in Polen war die Abschaffung der Zensur und die Aufnahme uneingeschränkter geschichtswissenschaftlicher Forschungen. Allerdings konzentrierten sich die Historiker hauptsächlich auf die Tilgung der sogenannten weißen Flecken in der Geschichte des Zweiten Weltkriegs (der Politik der UdSSR gegenüber Polen) und der Volksrepublik Polen, der Zeitraum zwischen 1918 und 1939 war bei den Forschern weniger beliebt.

Erst im zweiten Jahrzehnt des 21. Jahrhunderts wurde dieses Gebiet intensiver bearbeitet. Es lassen sich mehrere wichtige Forschungstrends identifizieren. Dies sind die Geschichte der Diplomatie mit dem wichtigsten Editionsprojekt der Polnischen Diplomatischen Dokumente ${ }^{2}$ sowie die Geschichte der nationalen Minderheiten - hauptsächlich der jüdischen, ukrainischen und deutschen. ${ }^{3}$ Besonders hervorzuheben ist die aktuelle Auseinandersetzung mit der Sozialgeschichte, vor allem das Projekt, das seit 2010 von der Abteilung für Sozialgeschichte des 19. und 20. Jahrhunderts des Historischen Instituts der Polnischen Akademie der Wissenschaften unter dem Titel „Gesellschaft der Zweiten Republik Polen. Versuch einer neuen Synthese“ durchgeführt wird. Im Rahmen dieses Projekts wurden bisher elf Bände zu verschiedenen Themen veröffentlicht. Ziel ist es, die Auseinandersetzung mit den Erkenntnissen der polnischen Sozialgeschichte der 1960er und 1970er Jahre mit aktuellen sozial- und geisteswissenschaftlichen Reflexionen zur Erforschung und Beschreibung von Sozialstruktur und sozialem Leben zu konfrontieren. Trotz des großen zeitlichen Abstands und des problemlosen Zugangs zu den Quellen wartet die Wirtschaftsgeschichte der Zwischenkriegszeit noch auf ihre Erforschung.

Mit der Gründung des polnischen Staats im Jahre 1918 begann ein langer Prozess des Aufbaus politischer, wirtschaftlicher und kultureller Strukturen. Es war eine sehr schwierige Aufgabe, nicht nur wegen der großen Zerstörungen der Kriege von 1914 bis 1921: Polen fußte infolge der Teilungszeit zunächst auf drei verschiedenen Staatsorganismen, die unterschiedliche Gesetze hatten, aber wirt-

\footnotetext{
${ }^{2}$ Vgl. www.pism.pl/publikacje/polskie_dokumenty_dyplomatyczne [4.2.2018].

${ }^{3}$ Für weitere Informationen zu dem Projekt vgl. www.ihpan.edu.pl/struktura/zaklad-historiispolecznej-xix-i-xx-wieku/ [4.2.2018].
} 
schaftlich eng mit den Märkten der Teilungsstaaten verbunden waren, welche sich nun aufgelöst hatten. Ein Symbol dieser Unterschiede war die Breitspurbahn, die im russischen Teilungsgebiet verkehrte. Nicht minder wichtig war die Frage der Nationalität. Nationale Minderheiten machten etwa ein Drittel der Bevölkerung des Lands aus.

Die Geschichte Polens in den Jahren von 1918 bis 1939 lässt sich in zwei Unterperioden unterteilen. Die erste umfasst die Periode zwischen 1918 und 1926 und war geprägt von einem Kampf um Grenzen sowie die Einführung der parlamentarischen Demokratie. Infolge des Staatsstreichs im Mai 1926 übernahm Józef Piłsudski, der von vielen Polen als Mitbegründer des unabhängigen Staats unterstützt wurde, die Macht in Polen, nachdem zuvor zahlreiche Regierungen einander abgelöst hatten. Anstatt jedoch die Demokratie zu sanieren (polnisch: sanac$j a$ ), wurde eine autoritäre Regierung gebildet. Sie dauerte bis zum Ende der Zweiten Republik Polen, also bis 1939. Piłsudskis Tod im Jahre 1935 bedeutete nicht ihr Ende, obwohl seine Nachfolger bei Weitem nicht an sein politisches Format heranreichten.

Die Einführung einer autoritären Regierung in Polen war nichts Besonderes. In vielen der damaligen Länder Mittel- und Osteuropas konnte man Ermüdung und Enttäuschung angesichts der ineffizienten, konfliktbeladenen parlamentarischen Demokratien beobachten. Viele Länder, mit Ausnahme der Tschechoslowakei, konnten der Versuchung nicht widerstehen, Regierungen der „starken Hand" einzuführen, von denen einige sich den faschistischen Diktaturen annäherten. Diese Länder wurden hauptsächlich vom Militär, von Teilnehmern des Ersten Weltkriegs, angeführt, die von Veteranen- und paramilitärischen Verbänden unterstützt wurden. Neben der Krise der parlamentarischen Demokratie haben die Wirtschaftskrise und die sich ständig verschlechternde soziale Situation den Ruf nach autoritären Herrschaftsformen verstärkt.

Piłsudskis autoritäre Regierung war relativ repressiv. Er eroberte die Macht durch einen Militärputsch, bei dem etwa 400 Menschen getötet wurden und später Vertreter der Opposition, die sich aus verschiedenen Bewegungen des nahezu gesamten politischen Spektrums zusammensetzten, im Lager Bereza Kartuska inhaftiert wurden. In der Politik orientierte er sich an der Idee eines starken und leistungsfähigen Staats, den er auch um den Preis der Demokratie zu schaffen bereit war, die er in der polnischen Version ohnehin nicht besonders schätzte.

Es besteht kein Zweifel, dass Piłsudski alle Merkmale eines charismatischen Politikers nach Max Weber besaß. Sein Tod 1935, kurz nach der Verabschiedung der neuen Verfassung, der sogenannten April-Verfassung, löste eine große Krise aus. Es setzte eine langsame Zerstörung des Herrschaftssystems ein, die politische und ideologische Rivalität der Nachfolger nahm zu. Streitigkeiten zwischen den verschiedenen Fraktionen des Regierungslagers und mit der Opposition standen auf der Tagesordnung und füllten die nächsten vier Jahre aus. Wie sich bald herausstellte, waren dies die letzten vier Jahre des Bestehens der Zweiten Republik.

„Bei Ausbruch des Kriegs gab es daher nicht nur politische Spaltungen zwischen der politischen Opposition, die durch die April-Verfassung und das undemokratische Wahlgesetz zu außerparlamentarischer Arbeit gezwungen wurde, 
sondern auch innerhalb des Machtlagers selbst“, schrieb Ryszard Kaczmarek, der Autor einer Geschichte Polens im 20. Jahrhundert. „Sie überlebten sogar die Zeit des Kriegs, sowohl in der Emigration als auch in Polen selbst, und wurden zum lästigen Ballast sowie zur Ursache des Zerfalls der antikommunistischen Opposition nach dem Zweiten Weltkrieg. “4

\section{Wieviel Zweite Republik gibt es in der Dritten Republik?}

Die Wiedererlangung der Unabhängigkeit Polens im Jahr 1989 war ein politischer und symbolischer Akt. Der neue Staat bezog sich bewusst auf die Zwischenkriegszeit und behandelte sich selbst als deren Fortsetzung. Dies spiegelte sich in der Verwendung verschiedener Symbole wider. Das neue Polen wurde Dritte Polnische Republik genannt, und der 11. November, der an die Erringung der Unabhängigkeit 1918 erinnert, (erneut) als Nationalfeiertag eingeführt. Am 15. August wird seitdem überdies der Jahrestag des Siegs der polnischen Truppen unter Führung Piłsudskis über die Rote Armee 1920 gefeiert, und der letzte Präsident der Republik Polen im Exil, Ryszard Kaczorowski, übergab seine noch aus der Zweiten Republik stammenden Insignien am 22. Dezember 1990 offiziell Präsident Lech Wałęsa.

Dies war eine weitere Bestätigung für die Verbindung mit der Zwischenkriegszeit. Die kommunistische Periode wurde damit über die Zäsuren der polnischen Geschichte hinaus verschoben, obwohl die Diskussion über Unabhängigkeit und Souveränität der Volksrepublik Polen noch andauert. Im öffentlichen Raum erschienen Denkmäler, die bis dahin verfemten, wichtigen Persönlichkeiten des 20. Jahrhunderts gewidmet waren, und Straßen wurden nach ihnen benannt. Es war auch von hohem symbolischen Wert, dass Gedenktafeln für die Kämpfe des Ersten Weltkriegs oder des polnisch-bolschewistischen Kriegs am Grabmal des unbekannten Soldaten wiederangebracht wurden, die die Kommunisten nach 1945 entfernt hatten.

Nach 1989 trat nur eine einzige politische Partei wieder auf den Plan, die sich unmittelbar auf die Tradition der Vorkriegszeit bezog, nämlich die Polnische Bauernpartei (Polskie Stronnictwo Ludowe). Die übrigen wichtigen Parteien wurden im Prinzip neu geschaffen, sie bezogen sich nicht direkt auf die Parteien der Zwischenkriegszeit. Die Reaktivierung der Sozialistischen Partei scheiterte, die parlamentarische Linke war postkommunistisch. Die nationale Bewegung, die sich auf die konservative Nationaldemokratie Dmowskis bezieht, blieb sehr lange am Rande, was nicht bedeutet, dass katholische und nationale Motive nicht in die Programme verschiedener rechtsgerichteter Gruppen aufgenommen wurden.

Die ersten Jahre nach der Wiedererlangung der Unabhängigkeit waren geprägt von tiefgreifenden politischen, wirtschaftlichen und sozialen Veränderungen. Die polnische Besonderheit bestand darin, dass diese Veränderungen durch einen Kompromiss zwischen den Machthabern und der antikommunistischen Opposition (Gespräche am runden Tisch, erste teilweise freie Wahlen zum Sejm im Juni

\footnotetext{
${ }^{4}$ Vgl. Ryszard Kaczmarek, Historia Polski 1914-1989, Warschau 2010, S. 368.
} 
1989) eingeleitet wurden. Bald darauf wurde dieser Prozess als „Refolution“5 bezeichnet, also ein revolutionärer Prozess mit einer evolutionären Vorgehensweise. Der Kompromiss mit den Machthabern wurde indes von einem Teil der früheren Opposition nicht mitgetragen, was bald darauf zur Spaltung und Radikalisierung der Positionen beitragen sollte.

Eine der Hauptaufgaben der neuen Regierung unter der Leitung von Premierminister Tadeusz Mazowiecki war die rasche Umsetzung der Reformen im Land und der Aufbau freundschaftlicher Beziehungen zu den Nachbarn. In vielen Fällen galt es, die Distanz und Vorurteile zu überwinden, die in der Zwischenkriegszeit entstanden waren, etwa in den Beziehungen zu Deutschland, der Ukraine, Tschechoslowakei und Litauen. Besondere Aufmerksamkeit wurde dem polnischjüdischen Dialog gewidmet. Einige dieser Aktivitäten waren eine Fortsetzung der Bemühungen, die während der Zeit des kommunistischen Regimes unternommen worden waren, manchmal im Gegensatz zur damaligen Regierung. Es war jedoch erst das freie Polen, das ihre ungehinderte Entwicklung ermöglichte, denn die polnischen Eliten hielten dies für einen notwendigen Bestandteil des Aufbaus des neuen Systems.

Es sei aber darauf hingewiesen, dass es keine öffentliche Debatte über die Zweite Republik Polen, die Schatten- und Glanzzeiten dieser Unabhängigkeit gab. Die Notwendigkeit einer solchen Auseinandersetzung wurde nur im Hinblick auf die Volksrepublik Polen anerkannt. Im Falle der Zweiten Republik Polen führte das allmähliche Verschwinden der Generationen, die sich an diese Zeit erinnerten, zu einem Mangel an gesellschaftlichem Druck für eine solche Debatte. Seitdem galt sie als eine Geschichte, die keinen Einfluss mehr auf die Gegenwart hat. Ihre Bedeutung bestand darin, der Dritten Republik Polen eine notwendige symbolische Dimension und einen historischen Kontext hinzuzufügen.

Die größte Herausforderung für die junge polnische Demokratie bestand darin, sich in europäische und atlantische Strukturen zu integrieren. Obwohl in den Debatten die Frage nach der Formulierung einer Politik gegenüber den östlichen Nachbarn wieder aufkam, in der die kritischen Bezüge zur Vorkriegszeit deutlich wurden, war der Marsch gen Westen das Wichtigste. Die politische, militärische und wirtschaftliche Integration mit ihm wurde als dauerhafter Schutz Polens vor der Einsamkeit und Bedrohung, in der sich Polen während der Zwischenkriegszeit befand, gesehen.

Die Erfolge des Transformationsprozesses und der Beitritt Polens zur NATO und zur EU haben Warschau zu einer führenden Kraft in Mittel- und Osteuropa gemacht. Die Präsenz Polens in den EU-Strukturen zu partnerschaftlichen Bedingungen wurde von der überwiegenden Mehrheit der polnischen Gesellschaft akzeptiert. Die konservativen Regierungen unter dem Banner der Partei Recht und Gerechtigkeit (Prawo i Sprawiedliwość; PiS) in den Jahren 2005 bis 2007 unterbrachen diese positive Tendenz nicht, aber es war ein Novum, dass einige Politiker der Regierungspartei direkt auf das politische Denken der Zwischenkriegszeit Be-

\footnotetext{
${ }^{5}$ Der Begriff stammt von Timothy Garton Ash, Ein Jahrhundert wird abgewählt. Aus den Zentren Mitteleuropas 1980-1990, München ${ }^{2} 1993$.
} 
zug nahmen. Formell wurde die Bereitschaft, das Vermächtnis von Piłsudski fortzuführen, erklärt, obwohl dieses eher vage definiert war, in der Praxis waren immer mehr die Ideen Dmowskis, des führenden Ideologen des polnischen Nationalismus, zu sehen.

Allmählich wurden jedoch immer mehr rechtsextreme Gruppen sichtbar, die sich auf Kräfte und Bewegungen in der Zweiten Republik Polen bezogen, gegen die die damaligen Machthaber repressive Maßnahmen ergriffen hatten. Die kritiklose Übernahme dieses Vermächtnisses und der Versuch, es unter den neuen Bedingungen zur Geltung zu bringen, hat jedoch Widerstand hervorgerufen, was den archaischen Charakter dieses politischen Ansatzes unterstrich. Das Motto des Tags lautete Europäisierung und Modernisierung. Offenbar gab es keine gesellschaftliche Nachfrage nach politischen Emotionen und radikalen ideologischen Programmen.

\section{Bitteres Material zum Nachdenken}

Die Situation änderte sich grundlegend, nachdem die PiS im Jahr 2015 wieder an die Macht kam. Die neue Regierung leitete radikale Veränderungen ein, die als Reformen bezeichnet und die unter der Losung „sich von den Knien erheben“ zusammengefasst werden. Trotz der vielen Proteste wurde die in der Verfassung festgeschriebene Gewaltenteilung rasch modifiziert (gelähmter Verfassungsgerichtshof, verstärkter Einfluss von Politikern auf die Wahl von Richtern und so weiter). Es wurde versucht, die Medien zu kontrollieren. Diese Veränderungen gingen Hand in Hand mit anderen. Eine Reform des allgemeinen Bildungssystems wurde eingeleitet; die Reform der Hochschulen steht kurz vor dem Abschluss. Auch ein affirmatives Bild der Geschichte Polens begann sich mehr und mehr durchzusetzen, was einen Bruch mit der bisherigen Politik bedeutet.

Im öffentlichen Raum werden die rechtsextremen Gruppen immer sichtbarer. Obwohl die PiS selbst nicht zu ihnen zählt, trug sie zweifellos zu ihrem Wachstum bei. Der Slogan, die „Pädagogik der Schande“ abzulehnen anstatt sich den dunklen Seiten der Geschichte zu stellen, führte dazu, dass eine oft nationalistische Vision der polnischen Vergangenheit vorherrscht.

Die Novellierung des Gesetzes über das Institut für Nationales Gedenken (Instytut Pamieci Narodowej; IPN), das Freiheitsstrafen wegen Verleumdung des guten Rufs Polens vorsieht, wurde verabschiedet. Wird es in diesem Jahr, dem Jahr des 100. Jahrestags der Wiedererlangung der Unabhängigkeit, in einer solchen Atmosphäre Raum für eine Debatte über die Zweite Republik Polen geben? Eine Diskussion über die Rolle von Dmowski und Piłsudski, die im November 2016 stattfand und die vom Komitee zur Verteidigung der Demokratie initiiert wurde, beweist ihre Notwendigkeit. ${ }^{6}$ Es ist bezeichnend, dass das virtuelle Pantheon, das damals

\footnotetext{
${ }^{6}$ Vgl. Gazeta Wyborcza vom 7.11.2016: „Kondzińska, Kijowski przeprasza za Dmowskiego i zaprasza na marsz KOD. Ale Szczuka na niego nie pójdzie“; www.wyborcza. pl / 7,75398,20943676, kijowski-przeprasza-za-dmowskiego-i-zaprasza-na-marsz-ale-szczuka. html [4.2.2018].
} 
geschaffen wurde, die Figur von Ignacy Daszyński, eines herausragenden sozialistischen Aktivisten und Premierministers, der bahnbrechende soziale und politische Reformen einleitete, ignorierte.

Trotz zahlreicher Proteste europäischer Institutionen und einzelner Staaten, mit denen Polen freundschaftliche Beziehungen unterhält, trotz des Widerstands einiger Teile der Gesellschaft, gibt die jetzige Regierung ihre umstrittenen Projekte nicht auf. Man könnte den Eindruck gewinnen, dass diese Veränderungen, „Deformierungen“, wie sie von Kritikern genannt werden, zur Befriedigung der eigenen Wähler durchgeführt werden. Die politischen und sozialen Kosten, die Auswirkungen auf die internationale Politik zählen nicht. Oftmals wird in den ÄuBerungen von Politikern der Regierungspartei der Wunsch nach einem starken Staat und damit nach einer Gemeinschaft von nationalen Polen betont. Sie beziehen sich auf Charaktere aus der Vergangenheit (stets nur Männer, was auch bedeutsam ist...).

Im vergangenen Jahr jährte sich zum 150. Mal die Geburt Józef Piłsudskis. Der Vorsitzende der PiS, Jarosław Kaczyński, hat bei dieser Gelegenheit einen offenen Brief an die Teilnehmer des Jubiläumskonzerts gerichtet. Er betonte darin, dass Piłsudskis historische Gestalt ihm und seinem verstorbenen Bruder, Lech, sehr nahestehe:

\begin{abstract}
„Wir sahen darin nicht nur eine der bedeutendsten Persönlichkeiten des europäischen politischen Lebens in den ersten Jahrzehnten des 20. Jahrhunderts und der bedeutendsten Polens, neben dem heiligen Johannes Paul II. und Kardinal Stefan Wyszyński. Wir haben uns auch - zum Teil natürlich unter grundlegend anderen Umständen - als Fortsetzer seiner Gedanken verstanden. [...] Es gab einige Differenzen zwischen uns, weil mein Bruder sehr vorsichtig mit der nationalen Tradition umgegangen ist, aber immer war, was sich in allen Programmdokumenten der Zentrumsallianz [Porozumienie Centrum] und PiS widerspiegelt, für uns das politische Denken in staatlichen Kategorien das Wichtigste.“7
\end{abstract}

Er verurteilte jedoch unmissverständlich die antidemokratischen Maßnahmen und forderte ein kritisches und differenziertes Urteil: „Die Machtübernahme mit Gewalt, das Heranführen des Staats an den Rand eines Bürgerkriegs ist inakzeptabel. Es ist auch unmöglich, die Prozesse von Brest, Bereza Kartuska oder andere Formen des Machtmissbrauchs nicht zu verurteilen."

Das ändert aber nichts an den Umständen, die die politische Landschaft seit der Machtübernahme der PiS in Polen radikal verändert haben. Nationalistisches und fremdenfeindliches Gerede wird im Namen der Slogans „gute Wende“ und „von den Knien aufstehen“ toleriert. Häufig verwendet die Regierungspartei populistische Parolen, die immer mehr Anhänger anziehen. Heute ist es schwierig

\footnotetext{
7 dziennik.pl vom 5.12.2017: „Kaczyński o Piłsudskim: ja i mój brat uważaliśmy się za kontynuatorów jego myśli“; www.wiadomosci.dziennik.pl/polityka/artykuly/564039,kaczynski-uwazasie-za-kontynuatora-mysli-pilsudskiego.html [4.2.2018].

${ }^{8}$ Ebenda.
} 
vorherzusagen, wie die Zukunft aussehen wird. Auf jeden Fall haben wir im Jahr des großen Jubiläums genug bitteres Material, um darüber nachzudenken. 